\title{
Color standardization method and system for whole slide imaging based on spectral sensing
}

\author{
Shinsuke Tani ${ }^{\mathrm{a}, *}$, Yasuhiro Fukunaga ${ }^{\mathrm{b}}$, Saori Shimizu ${ }^{\mathrm{a}}$, Munenori Fukunishi ${ }^{\mathrm{c}}$, Kensuke Ishiic and \\ Kosei Tamiya ${ }^{\mathrm{b}}$ \\ ${ }^{a}$ Olympus Corporation, Imaging Technology Department, Hachioji-shi, Tokyo, Japan \\ ${ }^{\mathrm{b}}$ Olympus Corporation, Imager \& Analog LSI Department, Hachioji, Tokyo, Japan \\ ${ }^{\mathrm{c}}$ Olympus Corporation of Americas New Business Research \& Development, Tokyo, Japan
}

\begin{abstract}
In the field of whole slide imaging, the imaging device or staining process cause color variations for each slide that affect the result of image analysis made by pathologist. In order to stabilize the analysis, we developed a color standardization method and system as described below.

1) Color standardization method based on RGB imaging and multi spectral sensing, which utilize less band (16 bands) than conventional method (60 bands).

2) High speed spectral sensing module.

As a result, we confirmed the following effect.

1) We confirmed the performance improvement of nucleus detection by the color standardization. And we can conduct without training data set which is needed in conventional method.

2) We can get detection performance of H\&E component equivalent to conventional method (60 bands). And measurement process is more than 255 times faster.
\end{abstract}

Keywords: Color standardization, color correction, dye amount, multi-spectral imaging, nucleus extraction

\section{Background}

In the field of whole slide imaging, the color appearance of tissue stained by $H \& E$ varies for each slide. These color variations affect the result of image analysis made by pathologists. Color standardization is important to stabilize the analysis.

These color variations are caused by not only by imaging device conditions but also staining conditions. Hence two kinds of approaches have been proposed to solve this problem. One approach is to calibrate imaging devices, cameras, and monitors to fit the color

\footnotetext{
*Corresponding author: Shinsuke Tani, Olympus Corporation, Imaging Technology Department, 2-3 Kuboyama-cho, Hachioji-shi, Tokyo 192-8512, Japan. Tel.: +81 42691 8073; Fax: +81 42691 7660; E-mail: shinsuke_tani@ot.olympus.co.jp.
}

property $[1,2]$. The other approach is to minimize the color differences caused by the staining process $[3,4]$. Previous research has exploited multi-spectral techniques to standardize color variations caused by the staining process [5]. There are a few problems with this method as described below.

a) Pathologists must make calibration slides using only one dye. These slides are not routinely made.

a) Previous research captured multi-band images using a crystal tunable filter. But the system takes 60 seconds to take 60 images.

In this research, we propose an advanced method that 1 ) does not require any calibration slides and 2) takes multi-spectral images quickly. We explain 2 techniques in sequence. 


\section{Color standardization method with estimation model for absorption spectra of dye}

\subsection{Introduction}

We developed a color standardization method based on multi-spectral techniques [1]. Our method consists of three processing steps as shown in Fig. 1.

Step1: Decompose H\&E stained image into hematoxylin and eosin components based on multi-spectral imaging techniques.

Step2: Adjust the ratio of each stain equal to that of a reference image.

Step3: Reconstruct corrected image from the adjusted components.

In our previous method the workflow is not practical for pathologists, because it is necessary for them to measure the absorption spectra using training samples stained by a single dye. Absorption spectra of dye vary with staining method, reagent, environment, and so on. And this difference has a large effect on colors as well as staining density. For example, in case of reagent, we can find the difference between stains by Muto Pure Chemicals and Merk as shown in Fig. 2. For getting the absorption spectra, it is necessary to make training samples stained using each dye separately and measure the absorption spectra using a 60 band multispectral sensor. These processes are too lengthy and inconvenient for real clinical use. In addition, we can't make training samples for specimens from the past or that have been discolored. We modified the algorithm to ease the pathologists as follows. We implemented the algorithm for estimating absorption spectra of dye from the multi-spectral signals of specimens. We propose an advanced method to improve the accuracy and flexibility of color standardization without training samples.

\subsection{Method}

We implemented the algorithm for estimating absorption spectra of dye from the multi-spectral images of specimens as shown in Fig. 3. For practically implementing our proposed algorithm, we detect

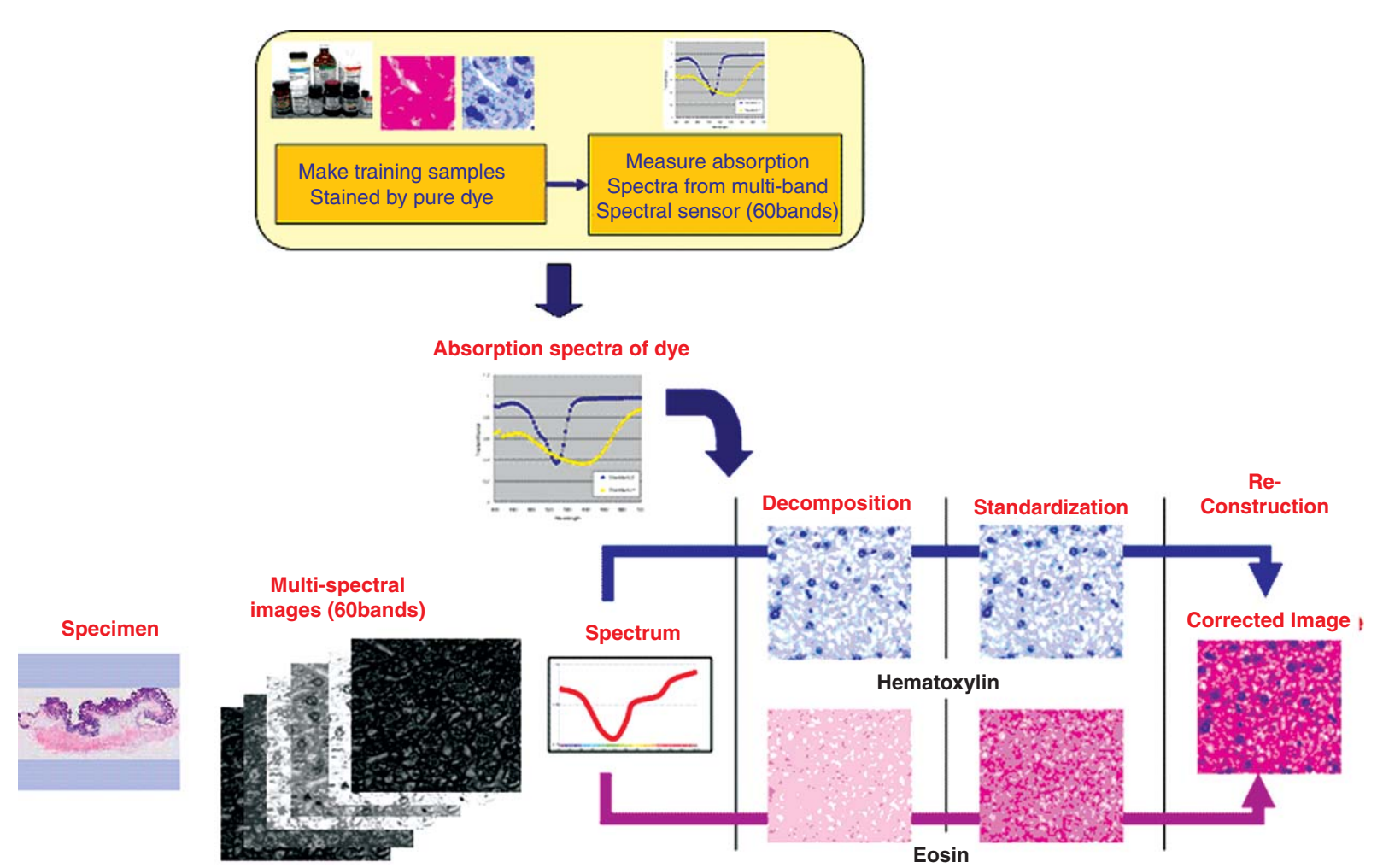

Fig. 1. Overview of the steps in the previous color standardization algorithm based on multi-spectral imaging techniques. 


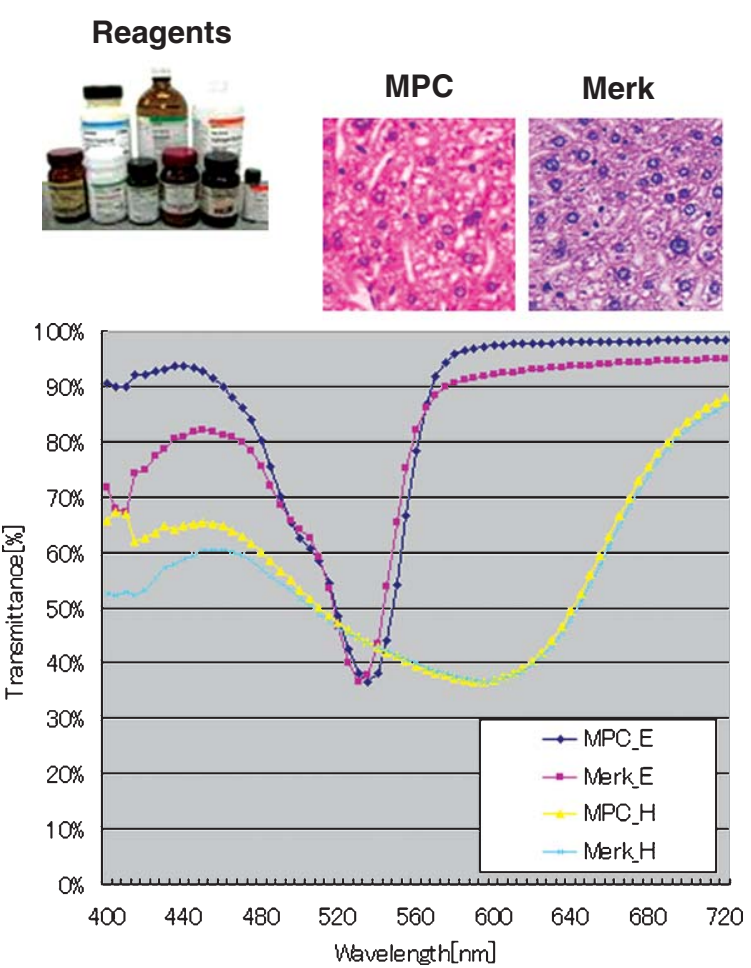

Fig. 2. Difference of transmittance spectra of dye (hematoxylin, eosin) between the reagent of MPC (Muto Pure Chemicals) and Merk.

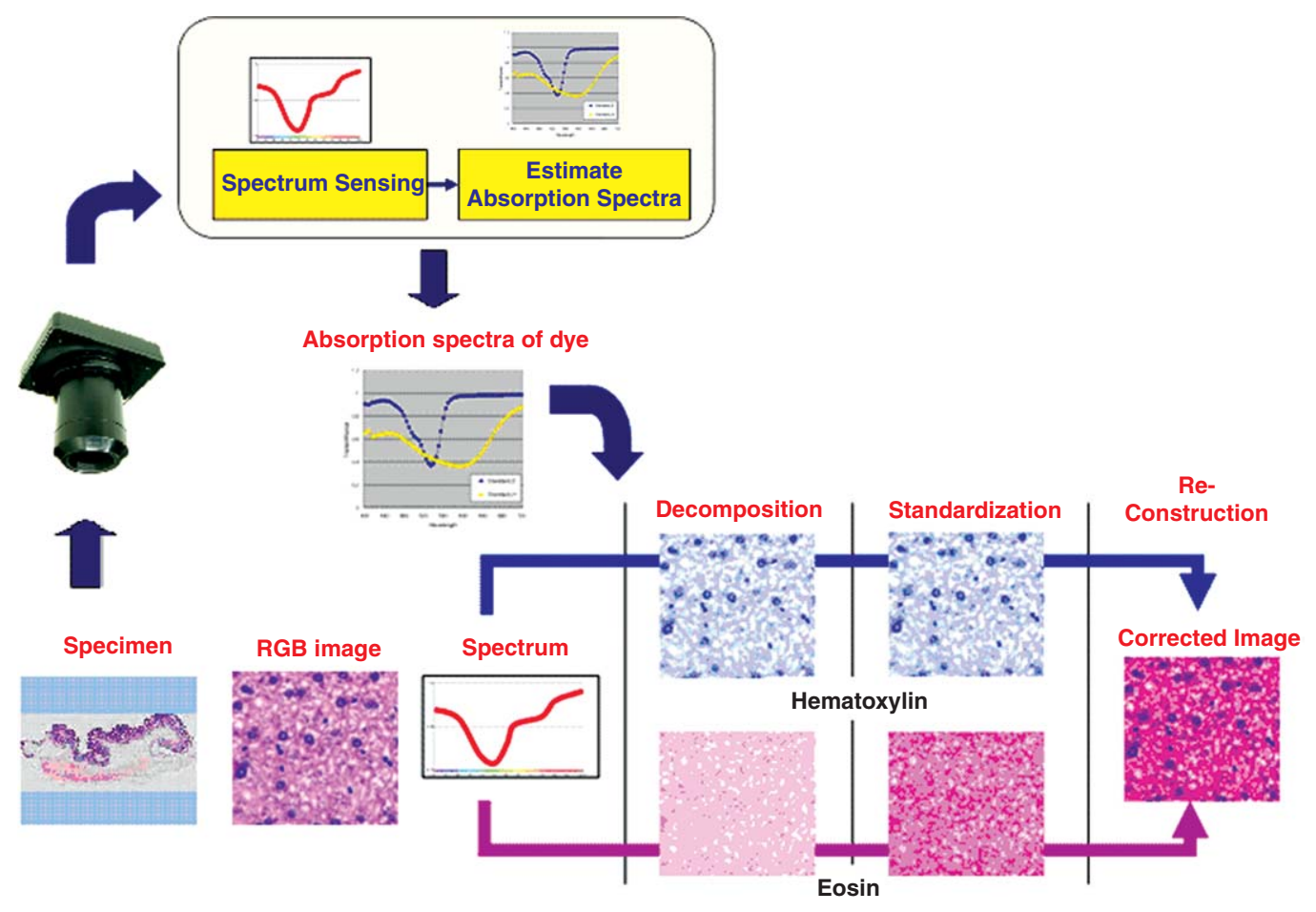

Fig. 3. Overview of the steps in the proposed color standardization algorithm. 


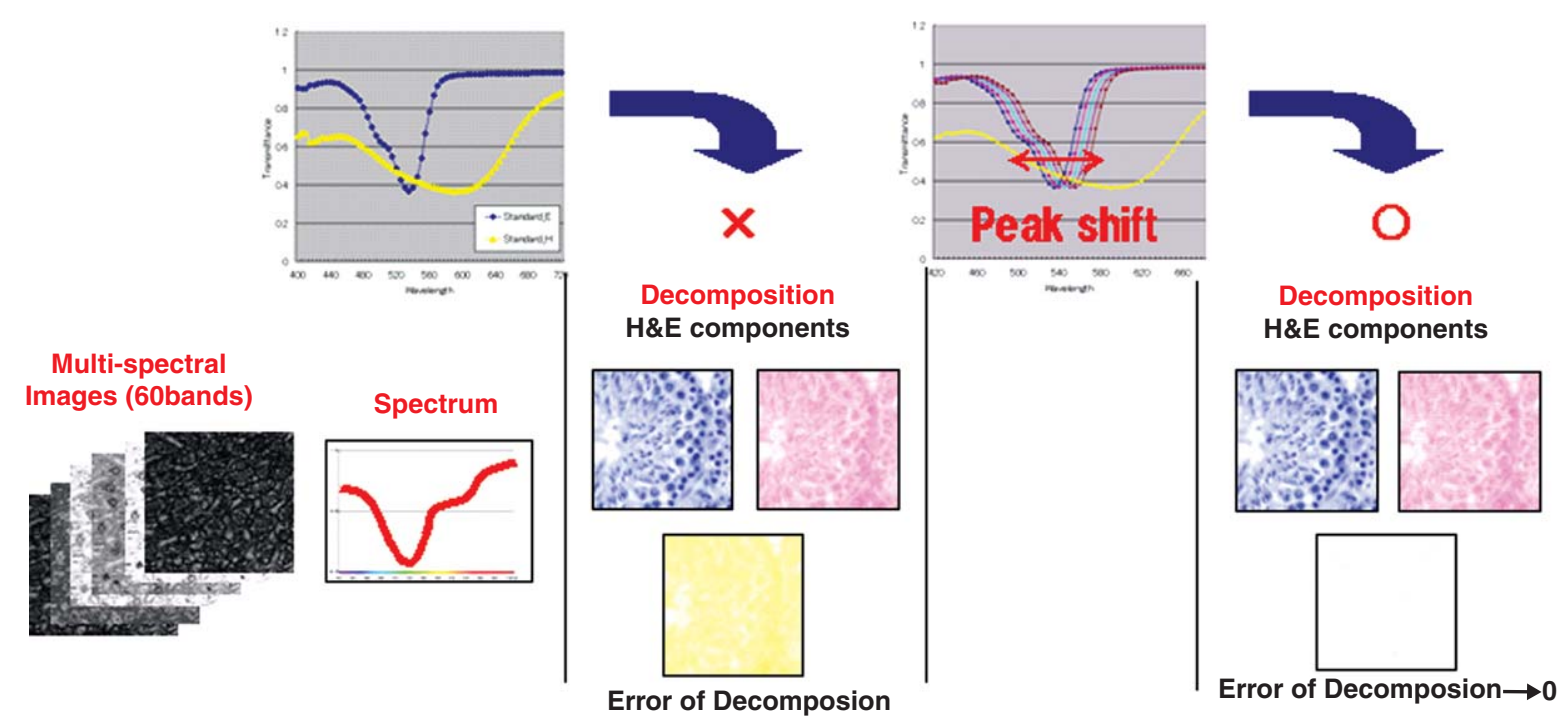

Fig. 4. Result of the proposed method for estimating absorption spectra of dye.
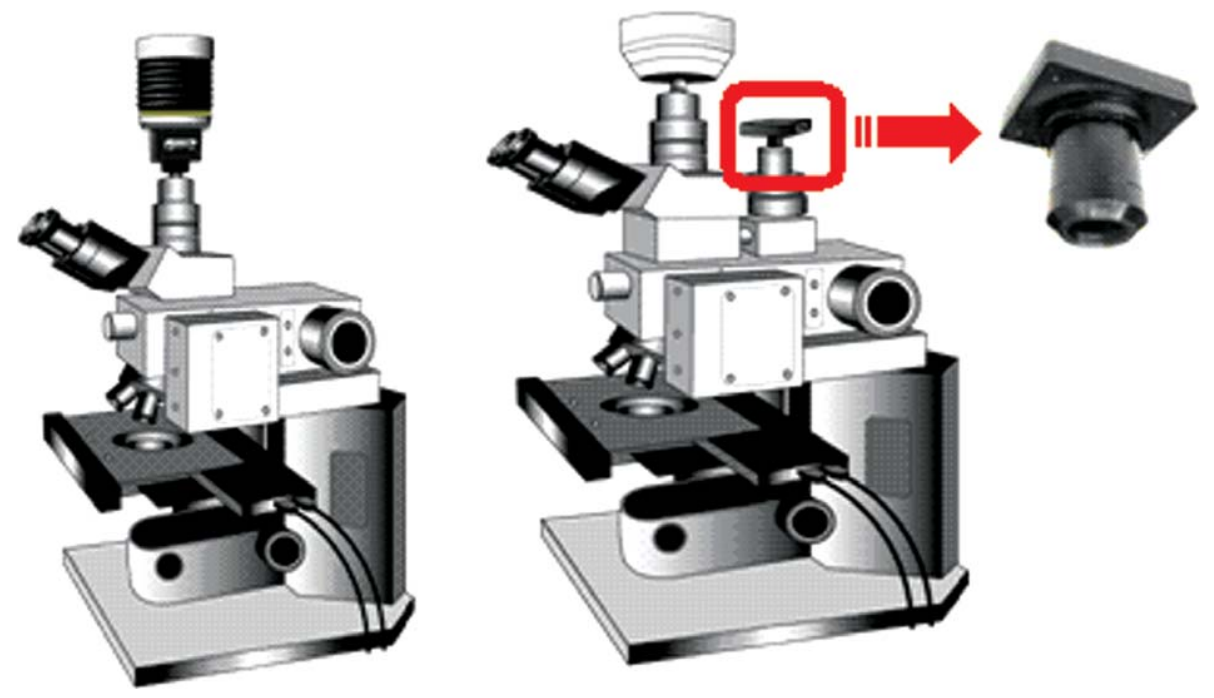

Fig. 5. Overview of experimental setup. The image on the left shows the previous setup for multi-spectral imaging using a standard microscope with a liquid crystal tunable filter attached at the top. The image on the right shows the proposed setup that uses an RGB sensor and a spectral sensor highlighted.

spectral signals with a spectral sensor and an RGB image using a standard microscope. The resultant system is more practical compared to the 60 band multi-spectral system. Our estimation method consists of the following steps. First, we extract and calculate the decomposition error as shown in Fig. 4. If decomposition error is 0 , the error image becomes white. Then we minimize decomposition error by optimizing the absorption spectra. We have prepared many models for optimizing, such as "Peak Shift", which shifts the peak of absorption of dye. For standardization, it is really important to replace absorption spectra of reference specimens using that of a target specimen.

Figure 5 shows the experimental setup. The previous method consists of a monochrome camera with a liquid crystal tunable filter that captures 60 bands images. The proposed method captures RGB images. Hence the proposed method does not need training samples. 


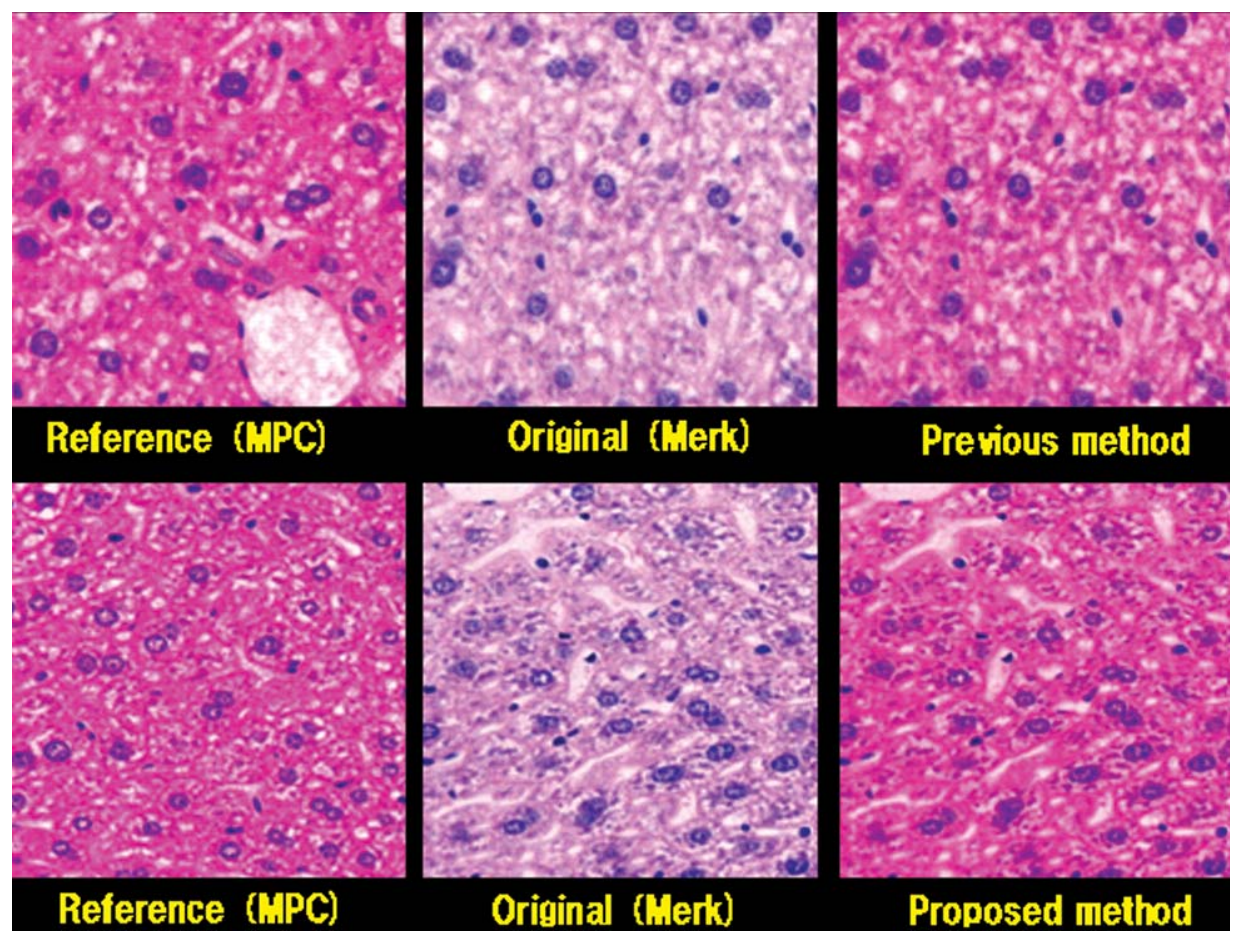

Fig. 6. Result of H\&E images. Images on the left are reference specimen. Images in the center are original specimen. Images on the right are the result of the color standardization methods. The top images are the result of the previous method. The lower images are the result of the proposed method. The results of the previous and proposed method are almost the same.

Table 1

Comparison of the experimental set up

\begin{tabular}{|c|c|c|}
\hline & Previous method & Proposed method \\
\hline Number of images & $\begin{array}{l}\text { Multi-spectral images } \\
\text { (60 bands) }\end{array}$ & $\begin{array}{l}\text { RGB image } \\
\text { (3 bands) }\end{array}$ \\
\hline $\begin{array}{l}\text { Special-purpose } \\
\text { device }\end{array}$ & LC tunable filter & Color sensor \\
\hline Speed & Slow & Fast \\
\hline Training samples & Need & No need \\
\hline
\end{tabular}

Table 1 shows the property comparison between the conventional and the proposed. We explain the detail of the color sensor in the next section ' 3 . Spectral sensing method for practical use'.

\subsection{Results}

We present the results by using the previous and proposed methods on processed H\&E specimens.

\subsubsection{Result of $H \& E$ images}

Figure 6 shows the result of H\&E images by using the color standardization algorithms to correct for different reagents. In this case, the proposed method achieved the same quality as the previous method and does not need training samples.

\subsubsection{Result of feature detection}

Figure 7 shows the result of using the algorithm of nucleus extraction. We utilized our ready-made product, "CellSens" and confirmed that its performance is improved by using the standardized image.

\section{Spectral sensing method for practical use}

\subsection{Introduction}

Multi-band cameras have been used for previous color standardization research. Figure 8 shows the microscope system equipped with a liquid crystal tunable filter and a monochrome camera. Table 2 shows a comparison between the previous and ideal measurements systems.

In our previous research, 60 minutes is needed to capture 60 band images. Each spectrum image is 


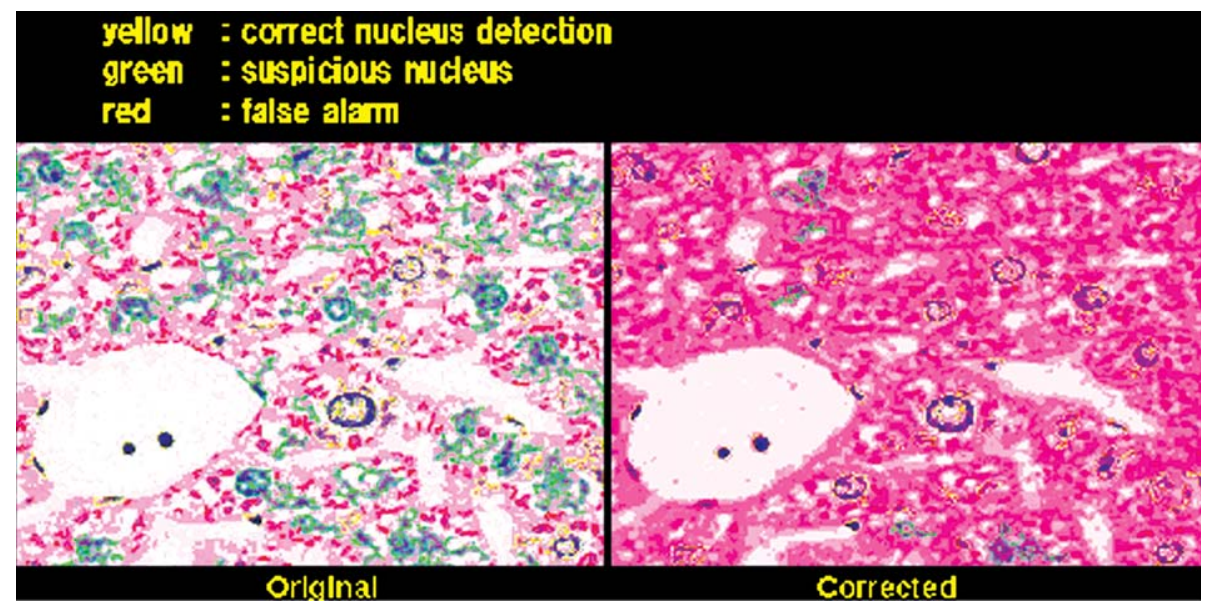

Fig. 7. Result of feature detection. The images on the left and right are the result of applying feature detection to the original and standardized images. In results from the corrected image, there are fewer nuclei outlined with green lines than the original image.

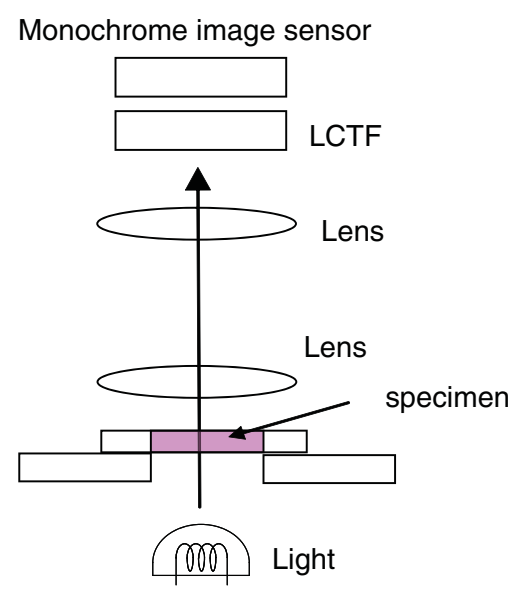

Fig. 8. Previous research system.

Table 2

Comparison previous research system with ideal system

\begin{tabular}{lll}
\hline & $\begin{array}{l}\text { Monochrome camera with } \\
\text { liquid crystal tunable filters } \\
(\text { LCTF)* }\end{array}$ & Ideal \\
\hline Speed & Slow & Fast \\
Number of images & 60 bands & 3 bands \\
Special-purpose device & LCTF & No need \\
Cost & High & Low \\
Size & Small enough & Small \\
\hline
\end{tabular}

*LCTF: Liquid Crystal tunable filter.

captured in series and mechanical tuning is needed before capturing one spectrum image. As a result, it is quite difficult to increase the speed of image capture.

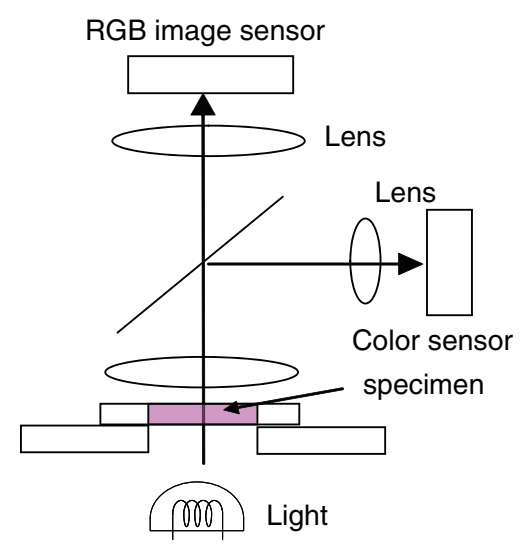

Fig. 9. Proposed research system.

\subsection{Method}

Figure 9 shows our new research system including a microscope, RGB camera, spectrum sensing unit and half mirror. The light from the transmitted specimen is divided into two by a half mirror. One is for RGB camera and the other is for the spectrum sensing unit. RGB camera and spectrum sensing unit operate independently, so the RGB camera and the spectrum sensing unit can operate on the same time.

Figure 10 shows spectrum sensing unit in detail. It is added to microscope and consists of optical aperture, light diffusion optical parts and $16 \mathrm{ch}$ active color sensor. 


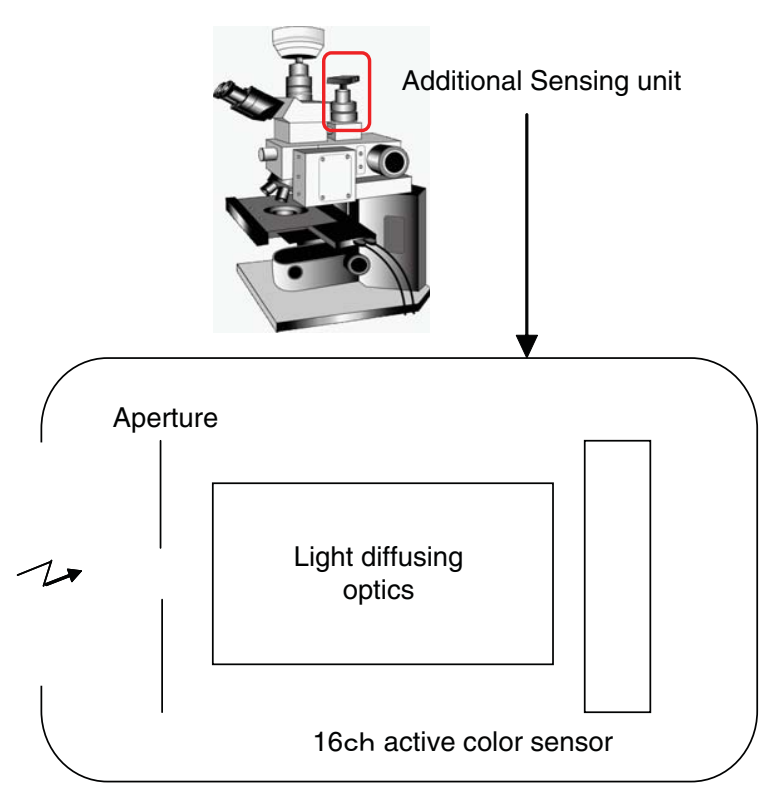

Fig. 10. Spectrum sensing unit.

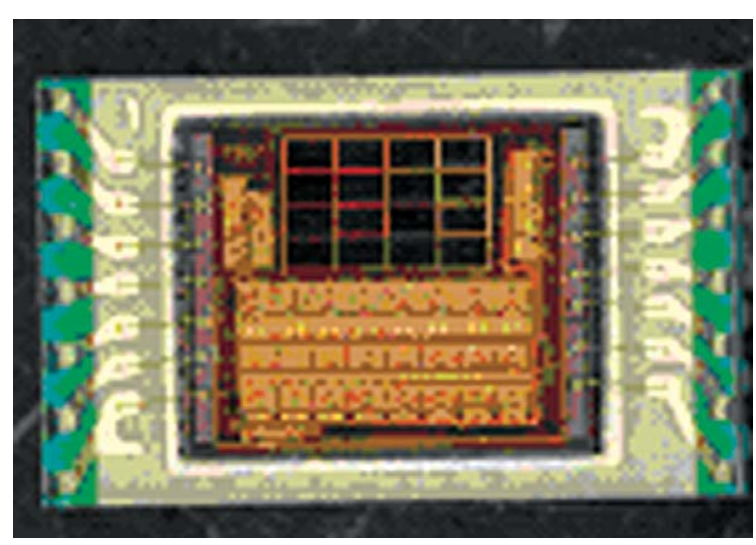

Fig. 11. Photo of $16 \mathrm{ch}$ active color sensor.

Figure 11 shows photo of $16 \mathrm{ch}$ active color sensor and Fig. 12 shows block diagram of it. It consists of 16 channel sensing unit and each sensing unit consists of color filter, photodiode and amplifier.

Figure 13 shows the relation to imaging area and spot of spectrum sensing. The imaging area is $0.44 \mathrm{~mm}$ $* 0.33 \mathrm{~mm}$ and diameter of sensing spot is $20 \mathrm{um}$. Spectrum sensing spot is smaller than imaging area. Imaging area and diameter of sensing spot are actual value when microscope optical power is 20 times.

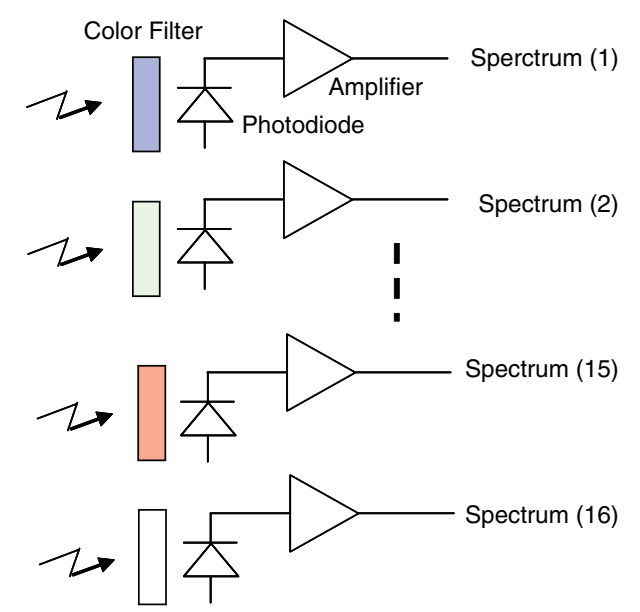

Fig. 12. 16 ch active color sensor in detail.

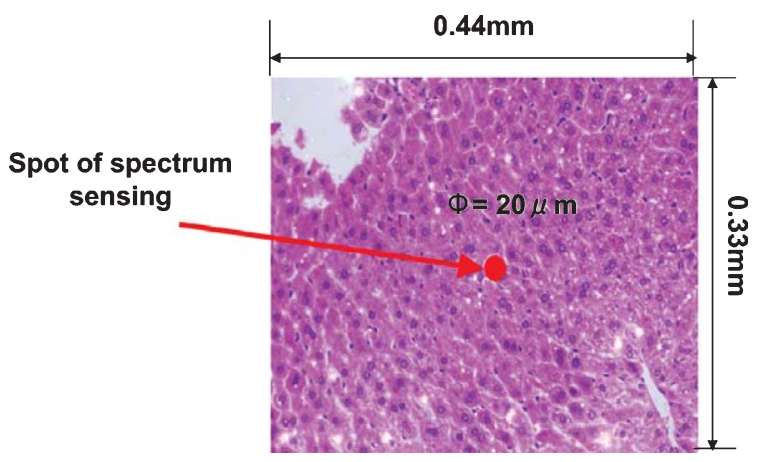

Fig. 13. Sensing size in specimen image.

Table 3

Experimental equipments

\begin{tabular}{ll}
\hline Item & Name \\
\hline Microscope & OLYMPUS BX52 \\
Light unit & OLYMPUS 12V100 W halogen bulb \\
RGB camera & OLYMPUS DP70 \\
Half mirror & OLYMPUS U-TR30* \\
\hline
\end{tabular}

$* 80 \%$ of light is for RGB camera and $20 \%$ of light is for spectral sensing in our research.

\subsection{Result}

The sensing time and quality were evaluated using a prototype. Table 3 shows the equipment we used for this research.

\subsubsection{Sensing time}

All outputs of $16 \mathrm{ch}$ Active color sensor are between noise and saturation when sensing time is $235 \mathrm{msec}$ and power of halogen lump is 225 lux. In the 


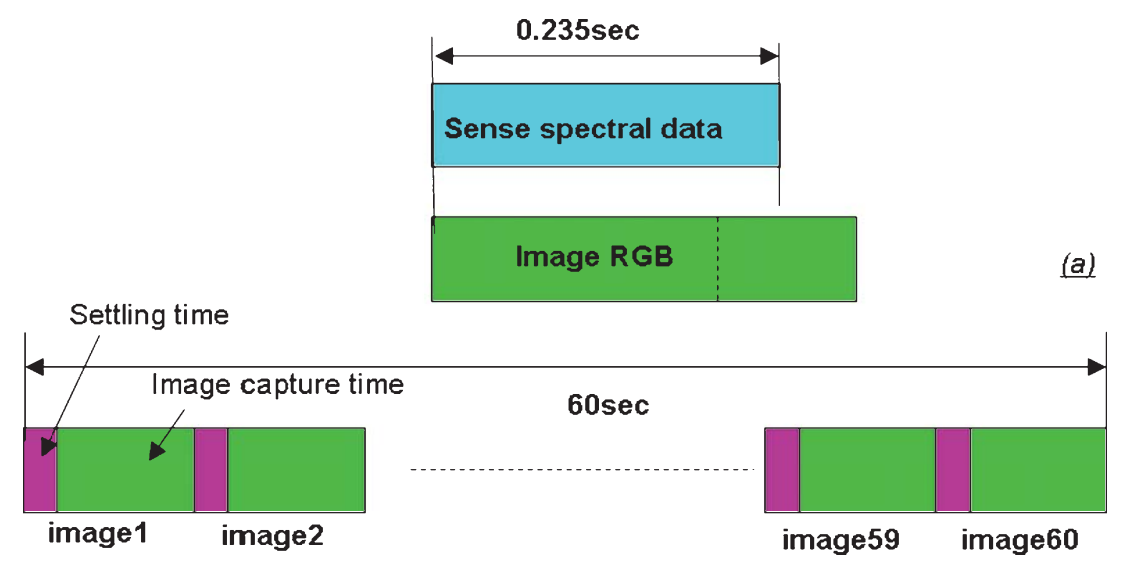

Fig. 14. Result of sensing time. Upper graph is the time chart of proposed system. Lower one is that of previous system.

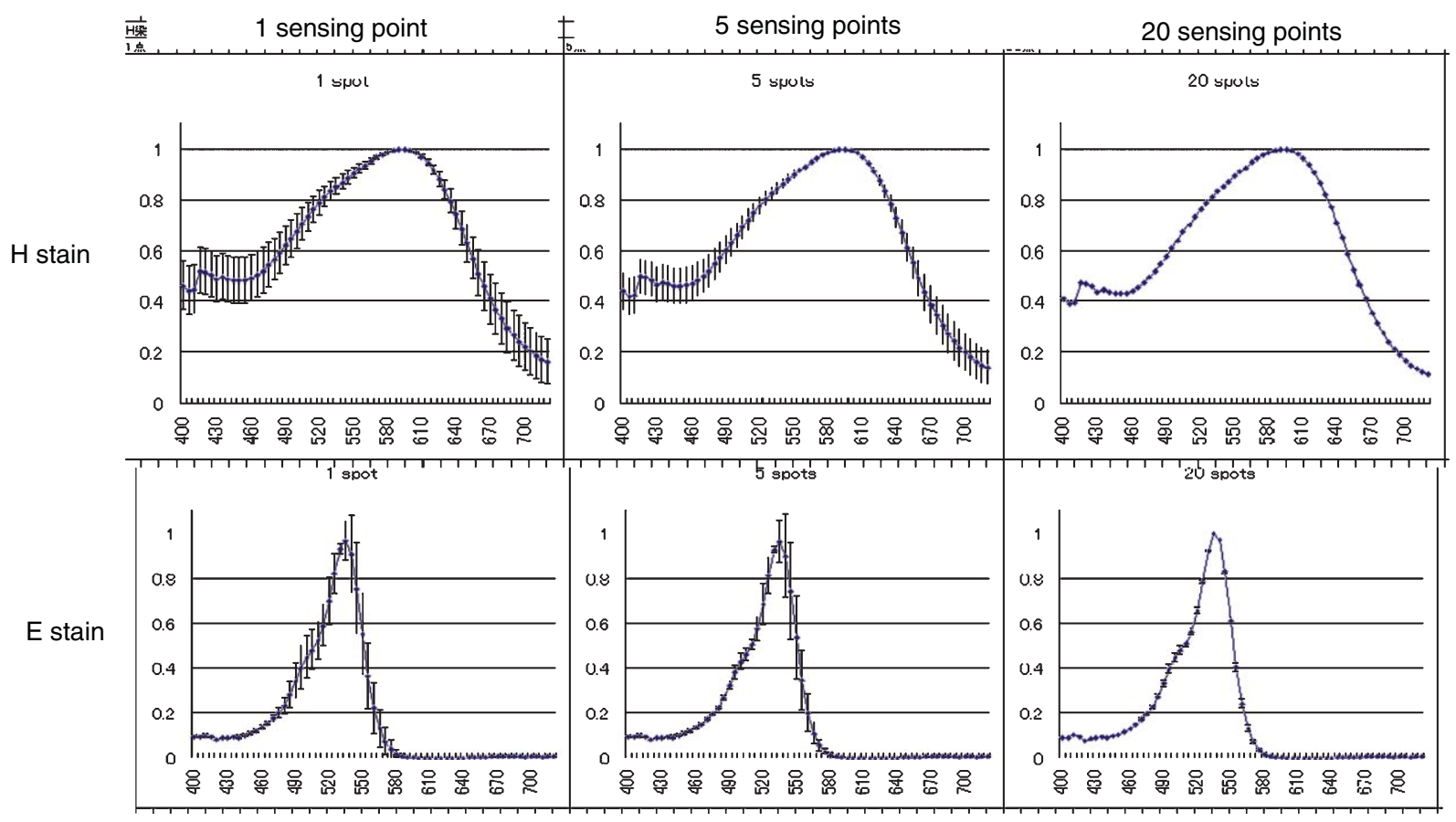

Fig. 15. Result of sensing quality I. Vertical axis is absorbance [a.u.] and horizontal axis is wavelength [nm].

previous research it takes $60 \mathrm{sec}$ to capture 60 multi band images. Figure 14 shows the comparison between previous research result and sensing time. Color sensor measured absorption spectrums of eosin \& hematoxylin within $235 \mathrm{msec}$. It is 255 times faster than the time to capture 60 images by LCTF.

\subsubsection{Sensing quality}

Figures 15 and 16 shows the estimated spectrum which is sensed by sensing spot shown in Fig. 13. Spot for spectral sensing is randomly selected on specimen. The left column is spectrum of hematoxylin and spectrum of eosin which is estimated from 1 sensing point. The center column is spectrum of hematoxylin and spectrum of eosin which is estimated from 5 sensing point. The right column is spectrum of hematoxylin and spectrum of eosin which is estimated from 20 sensing point.

As Fig. 15 shows sensed quality is increased according to the number of sensing points. Figure 16 shows 


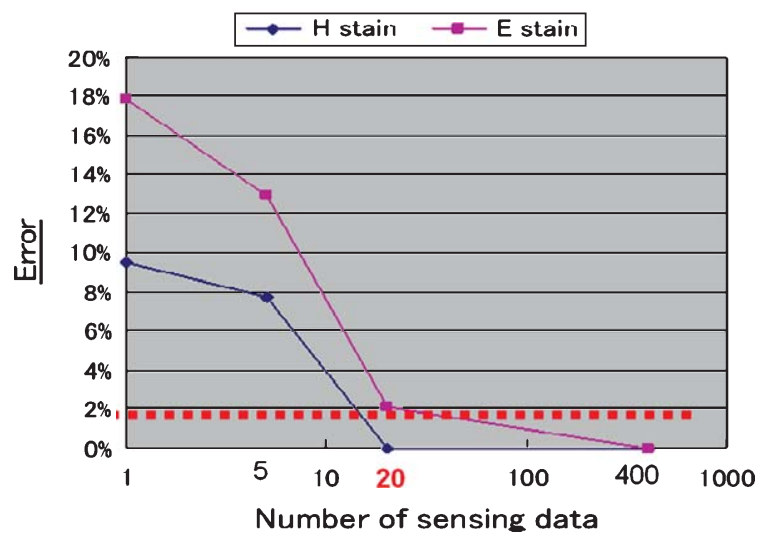

Fig. 16. Result of sensing quality II.

\section{Table 4}

Comparison proposed research system with previous research system. The first row is the previous research system. The second row is the proposed research system

\begin{tabular}{|c|c|c|c|}
\hline & $\begin{array}{l}\text { Monochrome } \\
\text { camera with LCTF }\end{array}$ & Our work & Ideal \\
\hline Speed & Slow & Fast & Fast \\
\hline Number of images & 60 & 3 & 3 band \\
\hline $\begin{array}{l}\text { Special-purpose } \\
\text { device }\end{array}$ & $\mathrm{LCTF}$ & Color sensor & No need \\
\hline Cost & High & Low & Low \\
\hline Size & Small enough & Small enough & Small \\
\hline Training sample & Need & No need & No need \\
\hline
\end{tabular}

quality of sensing according to sensing spot. $\mathrm{X}$ axis is number of sensing spot and $\mathrm{Y}$ axis is mean squared error: The reference spectrum is sensed by LCTF. The mean squared error of spectrum of hematoxylin and eosin are under $2 \%$ when number of sensing spot is more than 20 .

\section{Conclusions}

We proposed an advanced method of color standardization suitable for clinical use. And we experimentally show the effectiveness of the proposed method by successfully standardizing the color variation of $\mathrm{H} \& \mathrm{E}$ images without training samples. And our system is close to ideal as shown in Table 4 Our method is verified by a common microscope system.

In future work, we are planning to evaluate the effectiveness of the proposed method in a whole slide imaging system and to evaluate our color standardization for "tele-pathology" and "computer assisted diagnosis" for clinical use.

\section{References}

[1] Y. Yagi and J. Gilbertson, Digital imaging in pathology: The case for standardization, Journal of Telemedicine and Telecare 11 (2005), 109-116.

[2] Y. Yagi, Color standardization and optimization in whole slide imaging, Diagnostic Pathology 6(Suppl 1) (2011), S15.

[3] S. Arai, S. Kikuchi, Individual estimation of Hematoxylin and Eosin image distribution using a double-band microscope. EPOSTER, APIII (2003).

[4] T. Abe, Y. Murakami, M. Yamaguchi, N. Ohyama and Y. Yagi, Color correction of pathological images based on dye amount quantification, Optical Review 12(4) (2005), 293-300.

[5] T. Otsuka, H. Fukuda, P. Baytusta and Y. Yagi, Color Standardization, Session \#4 Color and Compression, Scientific Session, Pathology Informatics (2010). 


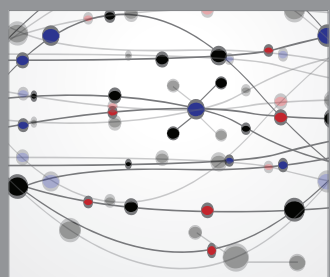

The Scientific World Journal
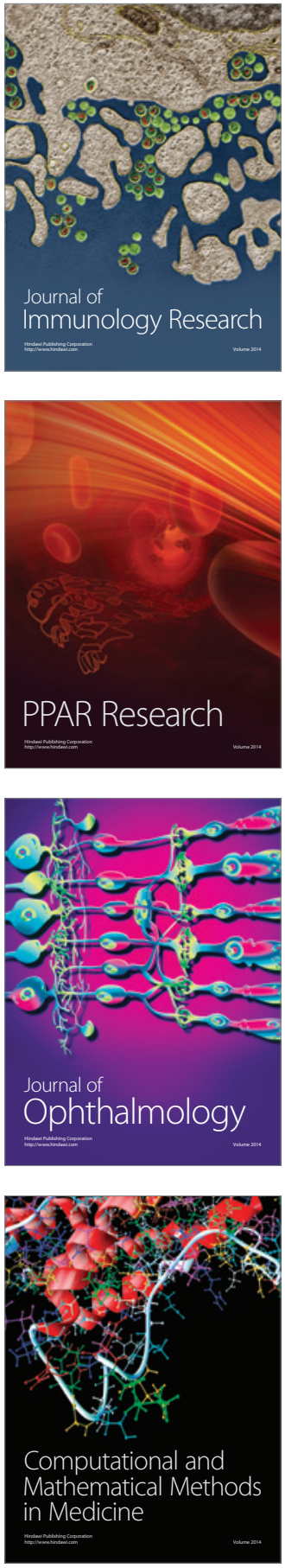

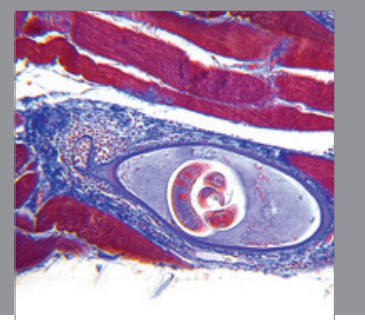

Gastroenterology

Research and Practice
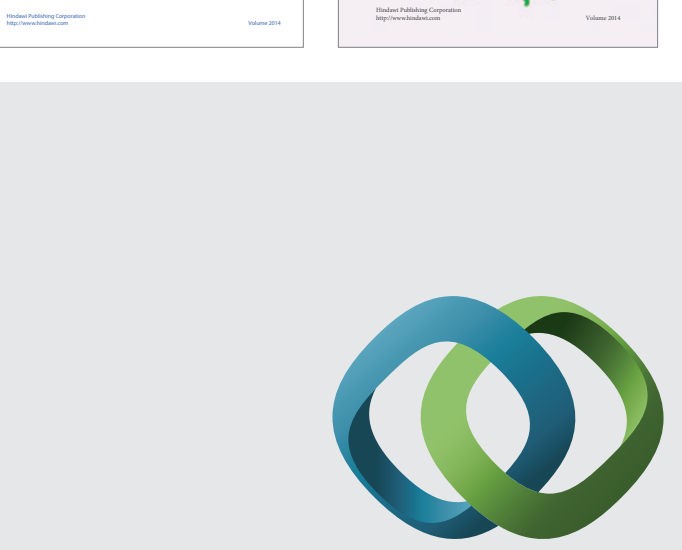

\section{Hindawi}

Submit your manuscripts at

http://www.hindawi.com
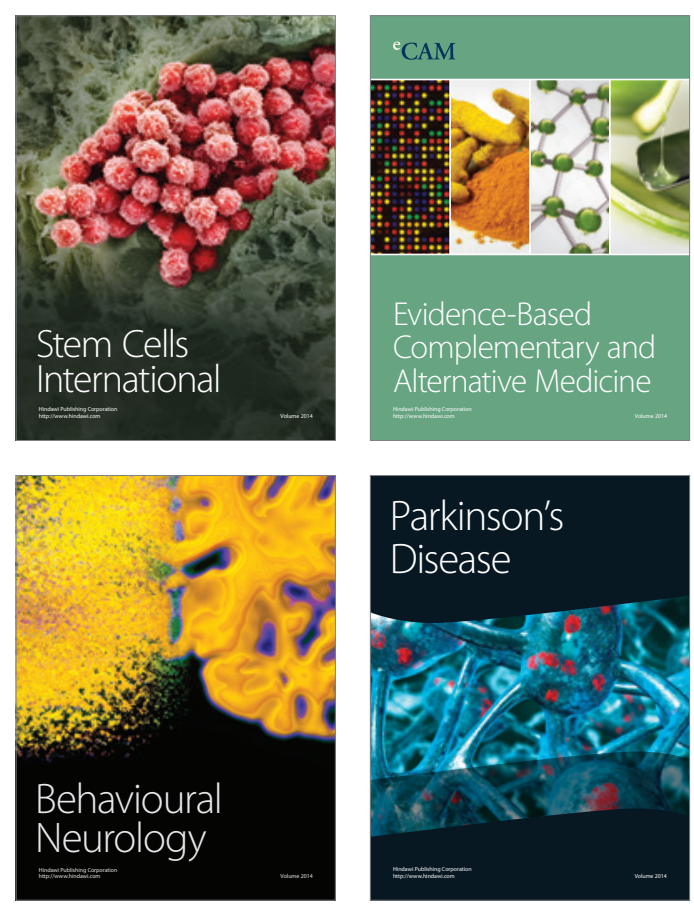

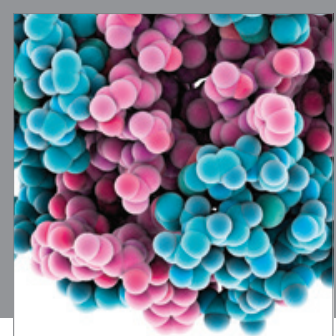

Journal of
Diabetes Research

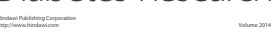

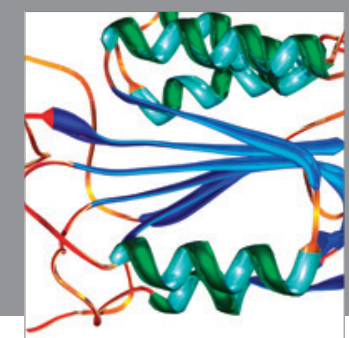

Disease Markers
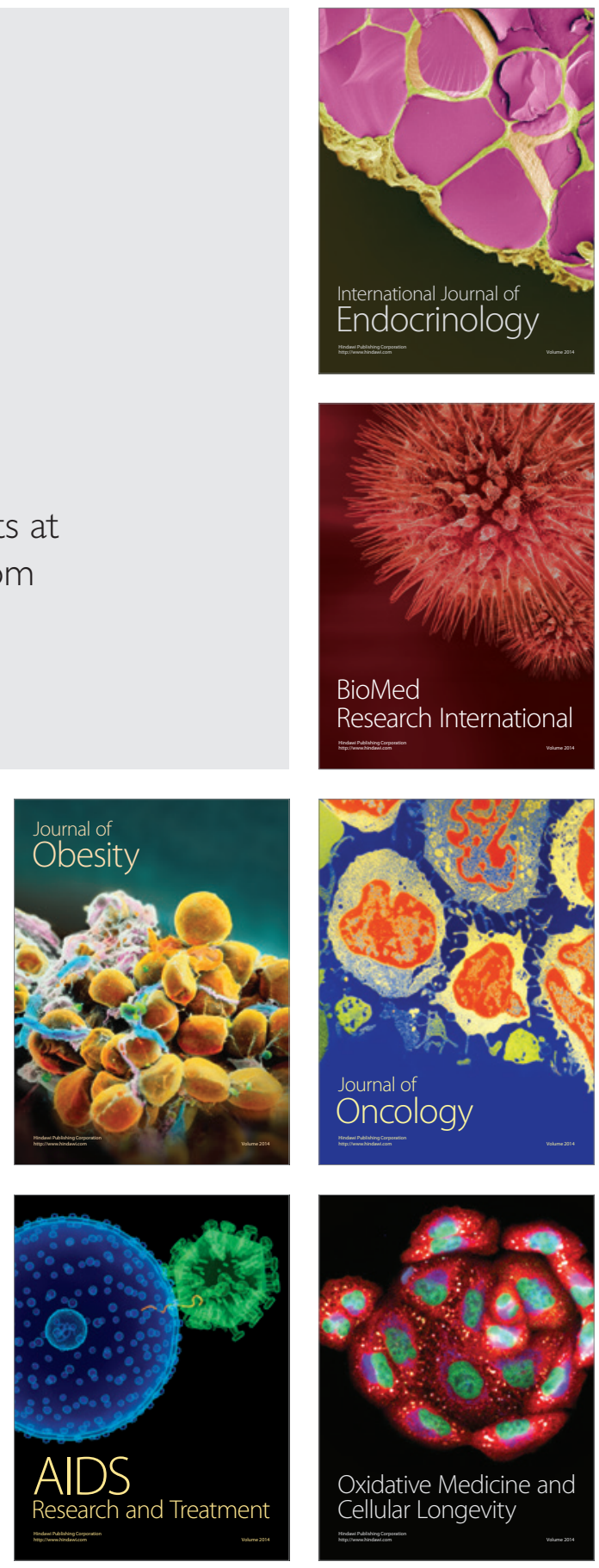